\title{
Análise de medicamentos administrados por sonda em unidades de terapia intensiva em hospital de ensino
}

\author{
Analysis of medications administered through tubes at an intensive care unit of a teaching hospital
}

Análisis de medicamentos administrados por sonda en unidades de terapia intensiva de hospital de enseñanza

Marcelo Rodrigues Martins ${ }^{1}$, Amanda Queiroz Soares², Ana Carolina Figueiredo Modesto³, Renata Figueiredo Carvalho ${ }^{4}$, Vivianne Vieira de Melo ${ }^{5}$, Izabel de Paula Duarte ${ }^{6}$

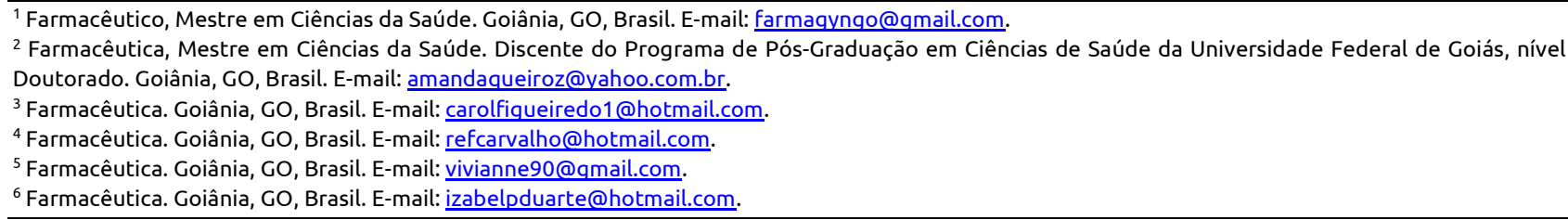

\section{RESUMO}

Este trabalho teve como objetivo analisar o uso de medicamentos administrados por sonda aos pacientes das unidades de terapia intensiva adulto e neonatal de um hospital de ensino. Trata-se de um estudo de caráter descritivo, exploratório e quantitativo, realizado em março/2011 a partir de prescrições oriundas das respectivas unidades, com foco nas recomendações de uso desses medicamentos via sonda. As informações foram coletadas e analisadas no programa Epi Info versão 3.5.2. Analisou-se 572 prescrições, somando 5.283 medicamentos. Dentre os medicamentos com forma farmacêutica de uso oral, a maioria (88,8\%) foi prescrita para administração por sonda. Desta, 11 tiveram restrição de uso. A alta frequência de medicamentos prescritos para administração por sonda, aliada aos diferentes tipos de restrições que inviabilizam seu uso por essa via, evidencia a necessidade da implantação de programas de educação continuada, bem como a criação de manuais educativos que busquem aprimorar o conhecimento dos profissionais envolvidos.

Descritores: Vias de Administração de Medicamentos; Prescrições de Medicamentos; Unidades de Terapia Intensiva; Nutrição Enteral.

\section{ABSTRACT}

The objective of this study was to analyze the use of medications administered though tubes to inpatients of the adult and neonatal intensive care units of a teaching hospital. This descriptive, exploratory, quantitative study was performed in March of 2011 based on prescriptions from the respective units, focused on the recommendations of the use of those medications through a tube. Information was collected and analyzed using Epi Info 3.5.2. A total of 572 prescriptions were analyzed, adding up to 5,283 medications. Among oral medications, most (88.8\%) were prescribed to be administered through a tube; and 11 were of restricted use. The high frequency of prescriptions for medications administered through tubes, associated with the different types of restrictions that make their use by this mean unfeasible, gives evidence of the need to implement continuing education programs, and to create educational handbooks seeking to improve the knowledge of the professionals involved.

Descriptors: Drug Administration Routes; Drug Prescriptions; Intensive Care Units; Enteral Nutrition.

\section{RESUMEN}

Se objetivó analizar la utilización de medicamentos administrados por sonda a pacientes de unidades de terapia intensiva adulta y neonatal de un hospital de enseñanza. Estudio descriptivo, exploratorio, cuantitativo; realizado en marzo de 2011 partiendo de prescripciones originadas en dichas unidades, haciendo foco en las recomendaciones de uso de tales medicamentos vía sonda. La información fue recolectada y analizada en el programa Epilnfo 3.5.2. Se analizaron 572 prescripciones, incluyendo 5.283 medicamentos. Entre los medicamentos de forma farmacéutica para uso oral, la mayoría (88,8\%) fue prescripta para administración por sonda. De ellos, 11 tenían restricciones para uso por esta vía. La gran cantidad de medicamentos prescriptos para administración por sonda aliada a las diferentes restricciones que desaconsejan su utilización por tal vía, expresan la necesidad de implantar programas de capacitación continua y la creación de manuales educativos que apunten a mejorar los conocimientos de los profesionales involucrados.

Descriptores: Vías de Administración de Medicamentos; Prescripciones de Medicamentos; Unidades de Cuidados Intensivos; Nutrición Enteral. 


\section{INTRODUÇÃO}

Nos Serviços Assistenciais à Saúde, a Unidade de Terapia Intensiva (UTI) se constitui como centro de monitoramento contínuo de pacientes em estado grave, com descompensação de um ou mais sistemas orgânicos, onde há possibilidade de restabelecer a homeostase, por meio de suporte altamente tecnológico e tratamento intensivo por equipe multiprofissional especializada ${ }^{(1)}$.

Dentre as atividades mais frequentes na prática diária da equipe assistencial, ações como a prescrição e a administração de medicamentos exigem conhecimento científico e habilidade técnica, para garantir a segurança na terapêutica medicamentosa administrada ao paciente $^{(1)}$.

No contexto assistencial da terapia intensiva, um aspecto que merece atenção é a administração de medicamentos por sonda aos pacientes em terapia nutricional enteral (NE). Este procedimento, quando realizado de forma inadequada, pode resultar na obstrução da sonda, na diminuição da eficácia do fármaco, no aumento de efeitos adversos ou na incompatibilidade do fármaco com componentes da $\mathrm{NE}^{(2)}$.

Com o intuito de evitar tais problemas, antes da administração dos medicamentos é necessária uma avaliação do ponto de vista farmacológico e farmacotécnico. No entanto, essa conduta é dificultada na prática clínica devido à escassez de informações encontradas na literatura sobre o tema, assim como nas especificações dos fabricantes de medicamentos ${ }^{(3)}$.

Apesar da responsabilidade pela administração de medicamentos por sonda ser primariamente da equipe de enfermagem ${ }^{(4)}$, todos os profissionais envolvidos no cuidado ao paciente precisam atentar-se para esse aspecto(5). Para tanto, é necessário a integração das ações da equipe multidisciplinar para possibilitar a identificação de possíveis fragilidades e falhas nos processos, promovendo intervenções que levem a melhorias visando a segurança do paciente ${ }^{(6)}$.

Diante da relevância do tema para a qualidade da assistência ao paciente, o presente estudo teve como objetivo analisar o uso de medicamentos administrados por sonda a pacientes das unidades de terapia intensiva adulto e neonatal, de um hospital de ensino, com foco nas recomendações de uso desses medicamentos por essa via.

\section{MÉTODOS}

Trata-se de um estudo descritivo, exploratório e quantitativo, desenvolvido no Hospital das Clínicas da Universidade Federal de Goiás (HC-UFG), uma instituição de ensino vinculado à Rede Sentinela do Ministério da Saúde. O hospital conta com aproximadamente 320 leitos, sendo 22 destinados a pacientes críticos: UTI Clínica (seis leitos), UTI Cirúrgica (oito leitos) e UTI Neonatal (oito leitos).

O processo de medicação no hospital em questão adota o seguinte fluxo: prescrição feita por um profissional habilitado, distribuição de medicamentos por dose individualizada pela Farmácia, levando em consideração os medicamentos padronizados na instituição, preparo e administração dos medicamentos pela equipe de enfermagem.

Foi considerado objeto do presente estudo as prescrições enviadas à Farmácia provenientes das três UTI's, no período de 01 a 31 de março de 2011. Foram incluídas todas as prescrições contendo medicamentos, e excluídos os seguintes itens prescritos: soluções parenterais de grande volume (SPGV), soluções de diálise e hemodiálise, itens de cunho não medicamentoso. É importante ressaltar que o presente estudo considerou como sonda de nutrição tanto sonda nasoentérica quanto nasogástrica.

A coleta de dados foi realizada no período de 01 a 30 de abril de 2011, com auxílio de um formulário estruturado desenvolvido para o estudo, contendo duas partes: 1) dados gerais da prescrição (nome do paciente, unidade clínica, data, quantidade de medicamentos prescritos por via de administração), 2) caracterização dos medicamentos administrados por sonda de nutrição (nome do medicamento, quantidade e forma farmacêutica). Os dados foram sistematizados e processados com auxílio dos programas Epilnfo versão 3.5.2. A análise estatística abrangeu determinação de frequência absoluta e relativa.

Vale ressaltar que o presente estudo foi submetido ao Comitê de Ética em Pesquisa do HC-UFG e aprovado sob número 180/2010, obedecendo aos princípios éticos da declaração de Helsinque e às normas legais da resolução 196/96 do Conselho Nacional de Saúde ${ }^{(7-8)}$. 


\section{RESULTADOS}

Durante o período do estudo foram analisadas 572 prescrições, as quais continham 5.283 medicamentos. Destes, 909 (17,2\%) eram medicamentos de uso oral, que puderam ser classificados em duas formas farmacêuticas distintas: 551 (60,61\%) sólidas e 358 (36,52\%) líquidas. A Tabela 1 apresenta a distribuição da frequência dos medicamentos prescritos de uso oral e formas farmacêuticas sólidas por unidade de internação.

Tabela 1. Medicamentos destinados à administração via oral de 572 prescrições médicas de três unidades de terapia intensiva do Hospital das Clínicas da Universidade Federal de Goiás, no período de 01 a 31 de março de 2011.

\begin{tabular}{ccccc}
\hline \multirow{2}{*}{ Unidade de Internação } & \multicolumn{2}{c}{ Medicamentos via oral } & \multicolumn{2}{c}{ Medicamentos sólidos orais } \\
\cline { 2 - 5 } & $\mathbf{n}$ & $\mathbf{\%}$ & $\mathbf{n}$ & $\mathbf{4}$ \\
\hline UTI cirúrgica & 236 & 25,96 & 235 & 53,65 \\
UTI clínica & 328 & 36,10 & 293 & 4,17 \\
UTI neonatal & 345 & 37,94 & 23 & $\mathbf{1 0 0}$ \\
\hline TOTAL & $\mathbf{9 0 9}$ & $\mathbf{1 0 0}$ & $\mathbf{5 5 1}$ & \\
\hline
\end{tabular}

Dos 909 medicamentos destinados à administração via oral, $806(88,8 \%)$ estavam prescritos para administração via sonda de nutrição. Destes, 327 (40,52\%) foram prescritos aos pacientes da UTI Clínica, 300
(37,17\%) aos da UTI Neonatal e 180 (22,30\%) aos pacientes da UTI Cirúrgica.

A Tabela 2 mostra a frequência dos medicamentos com formas farmacêuticas sólidas e líquidas prescritos para administração via sonda, por unidade de internação.

Tabela 2: Medicamentos sólidos e líquidos orais prescritos para administração por sonda em três unidades de terapia intensiva do Hospital das Clínicas da Universidade Federal de Goiás, no período de 01 a 31 de março de 2011.

\begin{tabular}{ccccc}
\hline \multirow{2}{*}{ Unidade de internação } & \multicolumn{2}{c}{ Medicamentos sólidos orais } & \multicolumn{2}{c}{ Medicamentos líquidos orais } \\
\cline { 2 - 5 } & $\mathbf{n}$ & $\mathbf{\%}$ & $\mathbf{n}$ & \% \\
\hline UTI cirúrgica & 180 & 37,58 & 05 & 1,49 \\
UTI clínica & 292 & 60,96 & 36 & 10,75 \\
UTI neonatal & 07 & 1,46 & 294 & 87,76 \\
\hline TOTAL & $\mathbf{4 7 9}$ & $\mathbf{1 0 0}$ & $\mathbf{3 3 5}$ & $\mathbf{1 0 0}$ \\
\hline
\end{tabular}

Dentre os medicamentos prescritos para formas farmacêuticas: solução oral, comprimido, cápsula administração por sonda de nutrição, verificou-se 57 e solução injetável. A Tabela 3 mostra a frequência destes diferentes medicamentos, contemplando as seguintes medicamentos.

Tabela 3: Medicamentos prescritos para serem administrados por sonda em três unidades de terapia intensiva do Hospital das Clínicas da Universidade Federal de Goiás, no período de 01 a 31 de março de 2011.

\begin{tabular}{ccc}
\hline Medicamentos & $\mathbf{n}$ & \% \\
\hline Polivitamínico solução oral & 71 & $8,86 \%$ \\
Anlodipino comprimido & 65 & $8,11 \%$ \\
Cálcio + vitaminas+ zinco solução oral & 56 & $6,99 \%$ \\
Sulfato ferroso solução oral & 53 & $6,61 \%$ \\
Clonidina comprimido & 49 & $6,11 \%$ \\
Ácido fólico solução oral & 48 & $5,99 \%$ \\
Domperidona solução oral & 44 & $5,50 \%$ \\
Paracetamol comprimido & 30 & $3,70 \%$ \\
Ácido acetilsalicílico comprimido & 28 & $3,50 \%$ \\
Outros* & 363 & $44,63 \%$ \\
\hline TOTAL & $\mathbf{8 0 7}$ & $\mathbf{1 0 0}$
\end{tabular}

*Estão incluídos todos os medicamentos prescritos para administração por sonda com percentual inferior a 3,5\%, independente da forma farmacêutica. 
O Quadro 1 apresenta os medicamentos prescritos para administração por sonda de nutrição identificados no estudo, que possuem restrição de uso por esta via.

Quadro 1: Medicamentos com restrições para serem administrados por sonda prescritos em três unidades de terapia intensiva do Hospital das Clínicas da Universidade Federal de Goiás, no período de 01 a 31 de março de 2011.

\begin{tabular}{|c|c|}
\hline Medicamentos & Motivo da restrição \\
\hline Amiodarona comprimido & Evitar devido à ausência de estudos ${ }^{(3)}$. \\
\hline Atenolol comprimido & Administração em conjunto com NE reduz a absorção(9). \\
\hline Bisacodil comprimido & Perda do revestimento entérico pela trituração ${ }^{(3)}$. \\
\hline Carbidopa + levodopa comprimido & Não deve ser triturado devido à possibilidade de obstrução da sonda (3). \\
\hline Fenitoína comprimido & $\begin{array}{l}\text { Administração em conjunto com NE reduz a absorção do fármaco devido à } \\
\text { complexação com íons }{ }^{(3,9)} \text {. }\end{array}$ \\
\hline Fenobarbital comprimido & $\begin{array}{l}\text { Evitar devido à ausência de dados específicos sobre a administração por } \\
\text { sondas }{ }^{(10)} \text {. }\end{array}$ \\
\hline Hidroclorotiazida comprimido & Administração em conjunto com NE reduz a absorção(9). \\
\hline Hidroxiuréia cápsula & Citostático, precaução para o manipulador ${ }^{(11)}$. \\
\hline $\begin{array}{l}\text { Nifedipino retard (liberação } \\
\text { controlada) comprimido }\end{array}$ & $\begin{array}{l}\text { Não deve ser triturado, devido à perda da característica de liberação } \\
\text { controlada, além do risco de obstrução da sonda }{ }^{(3)} \text {. }\end{array}$ \\
\hline Nimodipino comprimido & Administração em conjunto com NE reduz a absorção do fármaco ${ }^{(9)}$. \\
\hline Varfarina comprimido & $\begin{array}{l}\text { Pode ocorrer interação fármaco-nutriente com consequente diminuição da } \\
\text { sua concentração, principalmente com a vitamina } \mathrm{K}^{(3)} \text {. }\end{array}$ \\
\hline
\end{tabular}

Legenda: NE - Nutrição Enteral

\section{DISCUSSÃO}

A frequência de prescrição de medicamentos por via oral em pacientes internados em UTI's foi menor em comparação as demais vias de administração. No entanto, quanto aos medicamentos para administração oral, observou-se a predominância da forma farmacêutica sólida, o que condiz com dados encontrados na literatura. No entanto, esse achado se apresenta inconsistente quanto às recomendações de uso de medicamentos via sonda de nutrição, uma vez que as formas líquidas são preferíveis por essa via ${ }^{(12)}$.

A análise do perfil de prescrição das três UTI's incluídas no estudo demonstrou uma predominância de medicamentos prescritos pela forma farmacêutica sólida na UTI clínica, e um discreto destaque para medicamentos prescritos para administração via oral na UTI neonatal. Estes dados não foram confrontados com outras literaturas em virtude da ausência de resultados semelhantes.

Observou-se que os medicamentos que apresentaram maior frequência de administração por sonda são os que não apresentam restrições de uso por esta via, de acordo com a literatura pesquisada(3,9-10).

Quanto aos medicamentos que possuem restrições para administração por sonda de nutrição, faz-se necessário uma melhor compreensão dessas informações pela equipe multiprofissional para que a decisão pela utilização desses medicamentos por essa via seja adequada e segura.

Os medicamentos amiodarona e fenobarbital comprimidos não possuem investigações publicadas sobre a efetividade, segurança e alterações farmacocinéticas quando administrados por sonda de nutrição. Sendo assim, quando a via oral não for possível e/ou desejada, é preferível que outras formas farmacêuticas, sejam utilizadas como alternativa terapêutica ${ }^{(3,10)}$.

Em relação aos medicamentos atenolol, carbidopa+levodopa, fenitoína, hidroclorotiazida, nimodipino e varfarina, todos na forma de comprimido, recomenda-se que estes não sejam administrados via sonda concomitantemente à nutrição enteral, a fim de evitar a redução na solubilidade, bem como na absorção e concentração plasmática destes medicamentos ${ }^{(3,9,13)}$. Orienta-se, nestes casos, a interrupção da nutrição uma hora antes e duas horas após a administração dos medicamentos supracitados ${ }^{(12)}$.

Os medicamentos bisacodil e nifedipino retard apresentam restrição de administração via sonda de nutrição, em virtude das características farmacotécnicas desses comprimidos à trituração o que poderia implicar em: 1) destruição do revestimento que determina a 
liberação controlada do medicamento, no caso do nifedipino retard; 2) perda do revestimento entérico, no caso do bisacodil; 3) obstrução da sonda pelos excipientes farmacêuticos presentes na formulação dos comprimidos $(3,9,14)$.

Quanto à hidroxiuréia cápsula, recomenda-se que não seja administrada por sonda de nutrição, já que os aerossóis liberados pelo medicamento, quando há abertura da cápsula, podem expor os manipuladores a riscos devido ao potencial citotóxico que este fármaco apresenta. Em geral, medicamentos com potencial carcinogênico, teratogênico ou citotóxico não devem ser triturados (comprimidos) ou abertos (cápsulas) a fim de evitar riscos ao manipulador ${ }^{(11)}$.

Com a finalidade de contribuir com a qualidade da terapia medicamentosa com quaisquer medicamentos administrados por sonda de nutrição, alguns aspectos devem ser considerados: 1) evitar administração concomitante de medicamento com a nutrição; 2) interromper a dieta uma hora antes e duas horas após a administração do medicamento; 3) reajustar a bomba de infusão para o fornecimento das necessidades nutricionais totais para as $24 \mathrm{~h}$; 4) lavar a sonda antes e após a administração de cada medicamento; 5) evitar trituração de medicamento que apresente ação tóxica ao manipulador ${ }^{(11-12,15)}$.

Nesse contexto, a equipe de enfermagem deve estar atenta aos aspectos elencados anteriormente, uma vez que são os profissionais envolvidos em quase todas as etapas do processo de utilização do medicamento. Desta forma, evidencia-se a necessidade do profissional enfermeiro ampliar seus conhecimentos acerca da administração de medicamentos por sonda de nutrição, visando disseminar as informações a toda a equipe de enfermagem e garantir a efetividade do tratamento a que o paciente tem direito(16).

Além da equipe de enfermagem, todos os profissionais envolvidos nos cuidados ao paciente devem atentar-se sobre essa temática, a fim de evitar problemas relacionados à ineficácia dos medicamentos e garantir a segurança do paciente. A equipe multiprofissional de saúde, em especial enfermeiros, nutricionistas, médicos e farmacêuticos, deve ser estimulada a discutir a melhor conduta farmacológica em pacientes submetidos à sonda de nutrição(5).
Ao considerar a alta frequencia de medicamentos sólidos orais para administração via sonda de nutrição encontrados em nosso estudo, e a responsabilidade da equipe de saúde em garantir a segurança do paciente, torna-se evidente a importância da implantação de programas de educação continuada. Elaboração de manuais educativos que busquem aprimorar os conhecimentos desses profissionais sobre a administração de medicamentos via sonda de nutrição, também pode contribuir para ampliação do conhecimento acerca do assunto(4,17-18).

\section{CONCLUSÃO}

A alta frequência de medicamentos de uso oral prescritos para administração via sonda de nutrição observada neste estudo, predominando as formas farmacêuticas sólidas, vem chamar a atenção da equipe multiprofissional de saúde para este tema.

Em virtude dos diferentes tipos de restrições que inviabilizam o uso de alguns medicamentos por sonda, a equipe de saúde deve estar qualificada para identificar e intervir prontamente quando necessário, assegurando a qualidade da terapia medicamentosa.

Para otimizar a farmacoterapia e garantir a segurança do paciente, faz-se necessário melhorar a estrutura e o processo de uso de medicamentos administrados por sonda de nutrição.

Sendo assim, destaca-se a importância da implantação de programas de educação continuada, bem como a criação de manuais educativos que busquem aprimorar os conhecimentos dos profissionais envolvidos.

Além disso, os serviços de saúde devem disponibilizar medicamentos que supram as necessidades terapêuticas institucionais, em especial no que concerne a formas farmacêuticas compatíveis para uso por sonda de nutrição, assim como alternativas terapêuticas para os casos em que há restrição de uso por esta via.

Adicionalmente, há necessidade de adequação do sistema de distribuição de medicamentos, visando a unitarização das doses nas condições ideais. Porém, a existência de uma estrutura ótima não garante processos adequados, fazendo-se necessário, acima de tudo, um comprometimento de toda a equipe multiprofissional de saúde para que os resultados terapêuticos desejados sejam alcançados. 


\section{REFERÊNCIAS}

1. Rodrigues MCS, Oliveira, LC. Erros na administração de antibióticos em unidade de terapia intensiva de hospital de ensino. Rev. Eletr. Enf. [Internet]. 2010 [acesso em: 29 mar 2013];12(3):511-9. Disponível em:

http://www.fen.ufg.br/fen_revista/v12/n3/pdf/v12n3a14.pdf. 2. Williams NT. Medication administration through enteral feeding tubes. Am J Health Syst Pharm. 2008;65(24):2347-57. 3. Lima GL, Negrini NMM. Assistência farmacêutica na administração de medicamentos via sonda: escolha da forma farmacêutica adequada. Einstein. 2009;7(1 Pt 1):9-17.

4. Phillips N, Nay R. A systematic review of nursing administration of medication via enteral tubes in adults. J Clin Nurs. 2008;17:2257-2265.

5. Hoefler R, Júlia SV. Administração de Medicamentos por Sonda. Boletim Farmacoterapêutica. 2009;(03 e 04):1-6. 6. Oliveira RB, Melo ECP. O sistema de medicação em um hospital especializado no município do Rio de Janeiro. Esc. Anna Nery. 2011;15(3):480-489.

7. Williams JR. The Declaration of Helsinki and public health. Bulletin of the World Health Organization. 2008;86(8). 8. Resolução $n^{\circ} 196$, de 10 de outubro de 1996 . Aprova as diretrizes e normas regulamentadoras de pesquisas envolvendo seres humanos. Diário Oficial da União (Brasília). 1996 out 16. 9. Nascimento MMG, Ribeiro AQ. Compilação de base de dados com recomendações para administração de medicamentos via sonda enteral. R. Bras. Farm. Hosp. Serv. Saúde. 2010;1(1):1-24. 10. White R, Bradnam V. Handbook of Drug Administratrion via Enteral Feeding Tubes. Londres: Royal Pharmaceutical Society of Great Britain; 2007.

11. Thomson FC, Naysmith MR, Lindsay A. Managing drug therapy in patients receiving enteral and parenteral nutrition. Hosp. pharm. 2000;7(6):155-164.

12. Carvalho AMR, Oliveira DC, Martins BCC, Neto JEH, Vieira VMSF, Silva LIMM et al. Análise da prescrição de pacientes utilizando sonda enteral em um hospital universitário do ceará. R. Bras. Farm. Hosp. Serv. Saúde. 2010;1(1):1-24.

13. Ferreira TRAS, Reis AMM. Terapia nutricional enteral. In: Gomes MJVM, Reis AMM. Ciências Farmacêuticas: Uma Abordagem em Farmácia Hospitalar. São Paulo: Atheneu; 2000. 14. Aguas M, Marin OS, Farigola RM, Martí MRV, Aragonés IS, Casanovas LO et al. Ficha Farmacológica: Administración de medicamentos por sonda nasogástrica. Rev. Mult. Gerontol. 2009;19(2):87-89.

15. Goñi VR, Sánchez SL, Baztán IA, Asiain EMC. Administración de fármacos por sonda digestiva. Enferm intensiva.

2001;12(2):66-79.

16. Silva LD, Lisboa CD. Consequências da interação entre nutrição enteral e fármacos administrados por sondas: uma revisão integrativa. Cogitare Enfermagem. 2011;16(1):134-140. 17. Mota MLS, Barbosa IV, Studart RMB, Melo EM, Lima FET, Mariano FA. Avaliação do conhecimento do enfermeiro de unidade de terapia intensiva sobre administração de medicamentos por sonda nasogástrica e nasoenteral. Rev Lat Am Enfermagem [Internet]. 2010 [cited 2011 Abr 20];18(5):88894. Available from:

http://www.scielo.br/pdf/rlae/v18n5/pt 08.pdf.

18. Wohlt PD, Zheng L, Gunderson Sh, Balzar SA, Johnson BD, Fish JT. Recommendations for the use of medications with continuous enteral nutrition. Am J Health Syst Pharm. 2009;66(16):1458-1467.

Artigo recebido em 11/10/2011.

Aprovado para publicação em 13/08/2012.

Artigo publicado em 31/03/2013. 